\title{
BMJ Open Development of a discussion tool to enable well-being by providing choices for people with dementia: a qualitative study incorporating codesign and participatory action research
}

\author{
Dianne Patricia Goeman, ${ }^{1,2,3,4}$ Marissa Dickins, ${ }^{1}$ Steve lliffe, ${ }^{5}$ Dimity Pond, ${ }^{6}$ \\ Fleur O'Keefe ${ }^{1}$
}

To cite: Goeman DP, Dickins M, lliffe $\mathrm{S}$, et al. Development of a discussion tool to enable well-being by providing choices for people with dementia: a qualitative study incorporating codesign and participatory action research. BMJ Open 2017;7:e017672. doi:10.1136/ bmjopen-2017-017672

- Prepublication history and additional material for this paper are available online. To view please visit the journal (http:// dx.doi.org/10.1136/bmjopen2017-017672).

Received 8 May 2017

Revised 21 August 2017

Accepted 23 August 2017

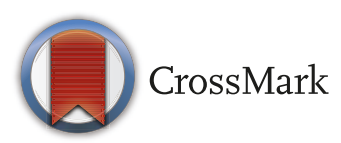

For numbered affiliations see end of article.

\section{Correspondence to} Dr Dianne Patricia Goeman; diannegoeman@yahoo.com; readdiannegoeman@yahoo.com

\section{ABSTRACT}

Objective To codesign a discussion tool to facilitate negotiation of risk between health professionals, people with dementia and carers.

Methods A qualitative approach using codesign. Thematic analysis was used to analyse interviews and focus groups with people with dementia, carers, healthcare staff and healthy older people exploring the issue of risk in dementia, the acceptability and development of a discussion tool.

Results Sixty-one participants identified the breadth, depth and complexity of risk in dementia care and the need for individualised solutions. They also deemed a discussion tool to facilitate negotiation of risk was acceptable and responses informed the tool development. Twenty-two participants provided feedback that was used to refine the final version.

Conclusion Our discussion tool enables choices for people with dementia by focusing on abilities rather than deficits and assists health professionals to deliver personcentred care. Flash cards prompt concerns and the tool provides a range of strategies to address these issues.

\section{INTRODUCTION}

Dementia is a progressive disease that can impact on one's ability to remember recent events, learn new skills, orientate to place and time and undertake activities of daily living. ${ }^{1}$ In the early stages of the condition, people with dementia may experience difficulties undertaking routine tasks, such as driving, shopping and managing their finances. As the disease progresses, self-care, bathing and eating may also pose problems. ${ }^{2}$ Consequently, people with dementia living in the community setting, and their carers and family, may benefit from both formal and informal supports and services to assist them to remain living in their own homes. ${ }^{3}$

Providing care for a person with dementia can pose a significant challenge to carers,

\section{Strengths and limitations of this study}

- Inclusion of codesign and participatory action research principles in the development of our discussion tool ensured that we explored the issue of risk from the perspectives of people with dementia, their carers, healthy older people, health professionals and healthcare organisations and that the tool was acceptable to each of these groups.

- To our knowledge, this is the first electronic resource that has been codesigned by consumers that enhances health professionals' ability to provide care according to the recently released Clinical Practice Guidelines and Principles of Care for People with Dementia by facilitating the delivery of 'Personcentred care', identifying and responding to the individual needs and preferences of the person with dementia, their carer and family.

- The development of an electronic discussion tool that can be incorporated into a health service organisations client record system for use as standard care for people living with dementia has provided a means and an evidence base on how such tools can be implemented across healthcare settings.

- Despite significant effort, the number of people with dementia recruited to this study was limited; however, appropriate numbers of carers were included which alleviates this concern to a small extent.

- The people with dementia who were included in this study tended to be younger and early in the trajectory of their disease and tended to reside in areas that are deemed as being higher socioeconomic status.

family and health and social care staff. Ethical dilemmas may arise in balancing the safety and well-being of a person with dementia and respecting their right to autonomy. ${ }^{4}$ For instance, family members and/or carers of people living with dementia are likely to be dealing with practical, day-to-day issues such 
as questioning whether going on an unaccompanied walk is desirable and feasible. ${ }^{5}$ Living with dementia is therefore a starting point for dealing with risk and awareness of the potential for future changes. For community dementia services to be responsive to the needs and wishes of their users, risk needs to be considered from within diverse local perspectives and life histories. ${ }^{5}$

The basic human right to autonomy is central to achieving high quality of life. ${ }^{6}$ This right to autonomy is reflected in the Australian Aged Care Act which specifically states that each person

'has the right to maintain his or her personal independence, which includes a recognition of personal responsibility for his or her own actions and choices, even though some actions may involve an element of risk where the resident has the right to accept, and that should then not be used to prevent or restrict those actions. ${ }^{7}$

This right to autonomy applies equally to people with dementia, including those who live in the community setting. Health professionals and health service providers remain risk averse despite the introduction of person-centred care and the aforementioned Aged Care charter and the Powers of Attorney Act, which states that a person is presumed to have decision making capacity unless there is evidence to the contrary. ${ }^{8-10}$ Frequently, there is pressure from healthcare professionals and concerned carers and family members to admit the person living with dementia to 'safe' and 'protected' residential care. This action is not necessarily safer and is frequently at odds with the wishes of the individual and the notion of 'person-centred care' and its guiding principle of putting people at the centre of their own care, including the provision of choice and control of their care services. ${ }^{911}$

A recent literature review that explored the issue of 'risk' in dementia care revealed a competing description of vulnerability and protection and a concern by health professionals about breaching their 'duty of care' or the threat of litigation undermining positive responses to risk. ${ }^{5}$ According to Ibrahim and Davis, ${ }^{10}$ there are four core systematic factors that impede the application of the 'dignity of risk' principle of allowing residents in the residential aged care sector the autonomy to accept risks that may be associated with short-term increases in their quality of life. These authors ${ }^{10}$ purport potential solutions to address this issue include: recognising that people with impaired cognition usually have some capacity for decision-making and expressing their preferences; seeking concordance between espoused values and actions and developing comprehensive accessible educational and point of care decision support tools for aged and healthcare providers. ${ }^{10}$

In the UK, the 'Dementia Strategy' recently released in England prioritises enabling people with dementia and their carers to live well with dementia in the community, ${ }^{12}$ with a focus on improving public and professional attitudes. ${ }^{13}$ Living well is broader than quality of life and is shaped by one's physical, social and cultural surroundings and the inclusion of life satisfaction which incorporates a sense of meaning and purpose in life. ${ }^{1415}$ As health professionals are increasingly expected to deliver person-centred care in an environment where there is potential for concern about the safety of their clients, there is recognition of the potential for the introduction of tools to support decision making that can alleviate professionals' anxiety. ${ }^{16}$

Currently in Australia however, there is little guidance to assist health professionals providing services that address client choices while mitigating unacceptable risk. To address this gap, we codesigned an electronic tool, with consumers and health professionals, that could facilitate a person's right to autonomy and assist health professionals and carers in the delivery of person-centred care. This paper reports on the experience-based codesign process and the development of an electronic discussion tool. ${ }^{17}$ In this instance, experience-based codesign involved users in the whole development process of the tool including assessment of its functionality and utility. ${ }^{18}$

The aim of our 'risk negotiation' discussion tool is to facilitate the well-being of people with dementia by enabling their choices, identifying concerns and providing strategies to address these and to document an agreed care plan.

\section{METHODS}

\section{Study design/theoretical framework}

The theoretical basis for the development of the 'risk negotiation' discussion tool followed a codesign and participatory action research (PAR) ${ }^{17} 18$ methodology to ensure that the issue of risk was explored from a range of perspectives. This included involving people with dementia, carers, healthy older people (older people without significant dementia experience; HOP), staff from a community nursing organisation and registered community nurses (RNs), alongside health professionals and healthcare organisations who provide support or healthcare to people living with dementia and their families (see section 2.7). The aforementioned participants were asked to inform the development of the risk negotiation tool that would facilitate discussion about risk and autonomy between people with dementia, carers/family and health professionals. Feedback from the participants was received in an iterative process that informed the selection of content and language included in the tool, refine the content and to evaluate the tools acceptability and utility to consumers and health professionals. The COREQ guidelines for reporting observational studies have been followed.

\section{Research team and reflexivity}

The research team was made up of a Senior Research Fellow (DPG) and a Research Fellow (MD), two academic general practitioners (SI and DP) and a Senior Clinical Nurse Advisor who has specialist training in aged care/ dementia (FOK). MD, a highly experienced qualitative 
Table 1 Participants

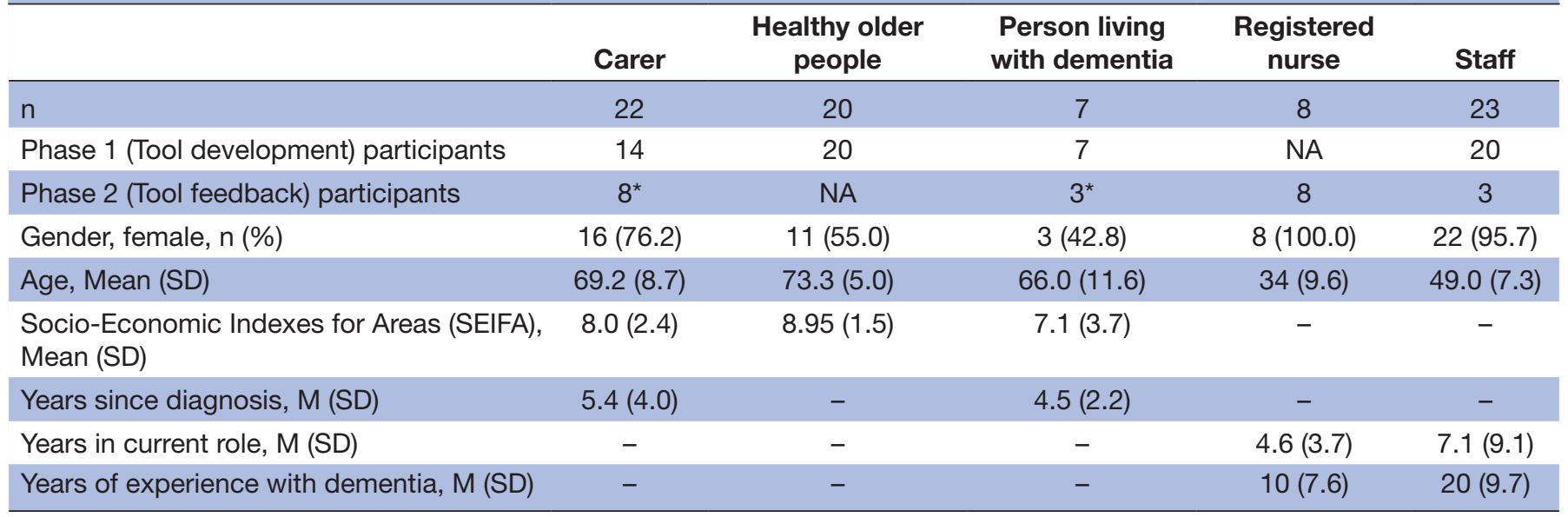

*Note some of these participants were interviewed in both exploration and feedback phases of the study.

researcher, undertook individual interviews with people with dementia, carers/family members, health professionals and healthcare organisation managers, FO completed interviews with people with dementia. DPG and MD facilitated the focus groups with HOP.

\section{Participant selection}

Purposive sampling was used to recruit all participants. Five groups of participants were recruited for this study: people with dementia, carers, HOP, RNs and staff from the community nursing organisation (comprising management, senior nurses and social workers).

Eighty-three interviews took place. Sixty-one interviews explored the issue of risk and autonomy and the development of a tool (Phase 1) and 22 interviews provided feedback on the tool postdevelopment (Phase 2) (see table 1). The expert stakeholder advisory group also provided feedback on the development and acceptability of the tool in an iterative fashion.

Participants were approached by MD, who provided them with written and verbal information about what would be involved in taking part in the study. Participants were asked to sign a consent form and consent was renegotiated at different points for participants with dementia to ensure that they were still agreeable to participating. Methods of approach included telephone, email and face to face contact.

\section{Data sources/study setting}

Face to face or telephone interviews were conducted with people with dementia, carers, RNs and staff. Focus groups were conducted with older people without significant dementia experience. Interviews took place at the home or the workplace of interviewees or other mutually suitable locations.

\section{Data collection}

There were two main phases involved in the development of the 'risk negotiation' discussion aid. Phase 1 involved interviews and focus groups with 61 participants exploring the overall issue of risk in dementia and specifically risk in the context of the provision of care to people with dementia. Each individual and focus group participant was asked during their interview about the risks that were associated with dementia and their views on a tool that would assist people with dementia, carers and health professionals to negotiate this issue in the context of dementia care.

After the completion of Phase 1, a draft tool was created based on the responses of participants. Phase 2 involved 22 interviews, with some participants from Phase 1 reinterviewed (eg, carers, people with dementia) and other participants (eg, RNs) invited to take part for the first time. Some carers came forward too late to take part in Phase 1, so took part in Phase 2 only. As part of this phase, all participants were provided with a demonstration of the aid and asked to provide feedback relating to modifications and its utility for use. New participants were also asked about their perceptions relating to the overall concept of risk in the context of dementia.

Demographic information was collected prior to each interview. All participants provided age and gender; carers and people with dementia also included year of diagnosis and service usage, while RNs and staff included information on current role and length of experience with dementia in a professional capacity. Postcodes for participants not being interviewed in a professional capacity (people with dementia, carers and HOP) were used in the present study as a proxy for socioeconomic status. Postcodes were then mapped onto the Index of Relative Socio-Economic Advantage and Disadvantage (IRSAD) based on postal areas, which ranks areas (eg, post codes) on a continuum from the most disadvantaged to the most advantaged (Australian Bureau of Statistics, 2013).

Interviews lasting between 11 and 76 min (mean $33 \mathrm{~min}$ ) were audiotaped and transcribed verbatim. Data collection continued until data saturation was reached. ${ }^{19}$ 


\section{Data analysis}

Thematic analysis was used to analyse the data in this study. Thematic analysis is a 'method for identifying, analysing and reporting patterns (themes) within data' (18, p. 79). Thematic analysis involves six phases: familiarisation, code generation, searching, review and theme naming and report production. These phases were used in this study and treated in a reciprocal manner, meaning that movement between the six phases occurred as required. An inductive strategy was used, allowing for themes to be generated from the data. Author 2 (MD) was primarily responsible for data analysis; however, regular discussions were held with the research team and expert advisory group as required to provide further insight into the themes arising from the analysis. NVivo V.11 was used to aid organisation of data and assist in analysis. ${ }^{20}$ Participants did not have access to the transcripts of their interview.

\section{Stakeholder advisory group}

The research team and 16 stakeholder advisory group members met quarterly (September 2015, December 2015, March 2016 and July 2016) prior to data collection and then throughout the duration of the 12-month project.

Members of the group, specifically chosen for their particular areas of expertise included: Consumers, Consumer Advocacy group representative/s, a Social Worker, a Mental Health Clinical Nurse Consultant, Health Service Quality and Risk Co-ordinators/Site Managers, a Human Rights Lawyer, a member of the Office of the Public Advocate, a Consultant Physician in Geriatric Medicine and expert in excellence in patient safety and a member of the Victorian Department of Health, Dementia and Support for Carers branch (see table 2). The group shared their understanding of the issues of risk and autonomy from their own perspective and also provided expert advice on:

- developing the project protocol,

- recruitment,

- appropriateness of materials/tools and resources,

- identification of issues or barriers that could impede the success of providing older people with greater choice and autonomy in decisions about their care and subsequently an improvement in their quality of life,

- overcoming problems with implementation of the project and

- discussing the key outcomes of the evaluation in order to identify any implications for further implementation of the negotiated risk agreement tool.

\section{RESULTS}

Of the 92 individuals who were invited to participate, 80 did so, resulting in an $87.0 \%$ participation rate. Of those invited that did not participate, four $(4.3 \%)$ declined participation, while eight $(8.7 \%)$ ceased responding to the attempts at contact made by the research team. While the majority of

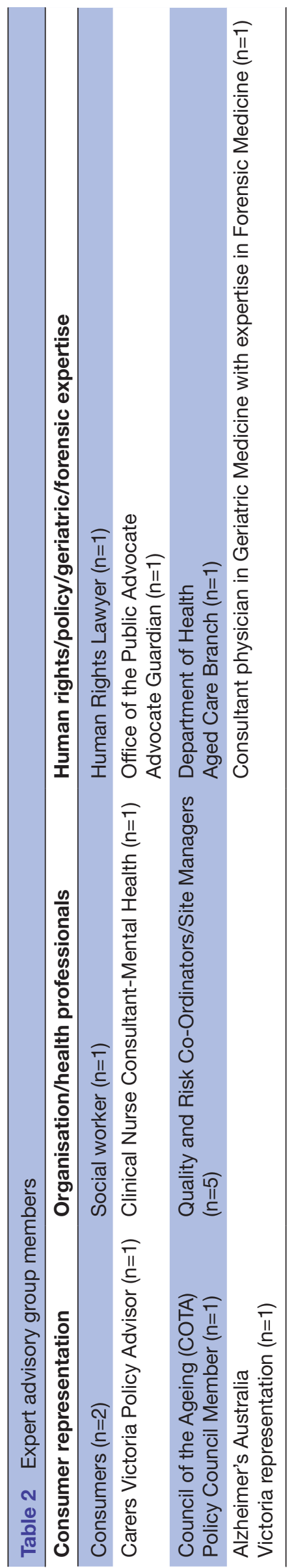


participants lived in Victoria $(\mathrm{n}=38,79.2 \%)$, some participants (people with dementia/carers) came from states other than Victoria (Queensland: $\mathrm{n}=4,8.3 \%$; Western Australia: $n=2,4.2 \%$; New South Wales: $n=1,2.1 \%$; the Australian Capital Territory: $\mathrm{n}=1,2.1 \%$; South Australia: $\mathrm{n}=1$, $2.1 \%$; Tasmania: $\mathrm{n}=1,2.1 \%$ ). The age of participants varied between 26 and 89 years $(\mathrm{M}=59.8, \mathrm{SD}=15.5)$, varying a great deal by participant type (see table 1 ).

In relation to socioeconomic status, the mean score of 8.0 and above for people with dementia, carers and HOP on the IRSAD indicates that in general, this group of individuals reside in areas considered to be of high socioeconomic advantage. The mean time since diagnosis recorded for both carers and people living with dementia was approximately 5 years. Those working as RNs had been in their current role for approximately 4.5 years and had 10 years of professional dementia experience. Those working as staff members had been in their current role approximately 7 years and had 20 years professional and/ or clinical dementia experience.

\section{Phase I: Exploration of risk and response to the development of a tool to negotiate risk}

Exploration of risk from the perspective of consumers, carers, health professionals and healthy older people

Overall nurses (both in management and those involved in direct clinical care), people living with dementia and carers (see table 1) had a fairly open view to those living with dementia taking 'acceptable risks'. One group of HOP was more risk averse and did not want the person with dementia taking any risks; however, the following two groups of HOP were open to the idea of 'acceptable risk' for people with dementia. While 59 different risks were identified (see figure 1) only seven risks were identified by a least one participant in each of the four groups. These risks were cooking, medication management, falls, driving, behavioural and psychological symptoms of dementia, social isolation and dressing. For other identified risks, see figure 1 .

Acceptability, utility and format of a 'Negotiated Risk Decision Aid' tool We asked people with dementia, carers and staff, their views on the acceptability, utility, format and implementation of a tool that would assist people with dementia, carers and health professionals to negotiate the issue of risk in the context of dementia. While participants believed a tool would be useful, they emphasised the need for the tool to be exploratory:

'... If possible [identify] what the person's lifestyle and choices were prior to them having dementia...I don't think it's about identifying what the risk is...It's just about what the choices are that they need ... so it's got to be an exploratory discussion' (Staff 002)

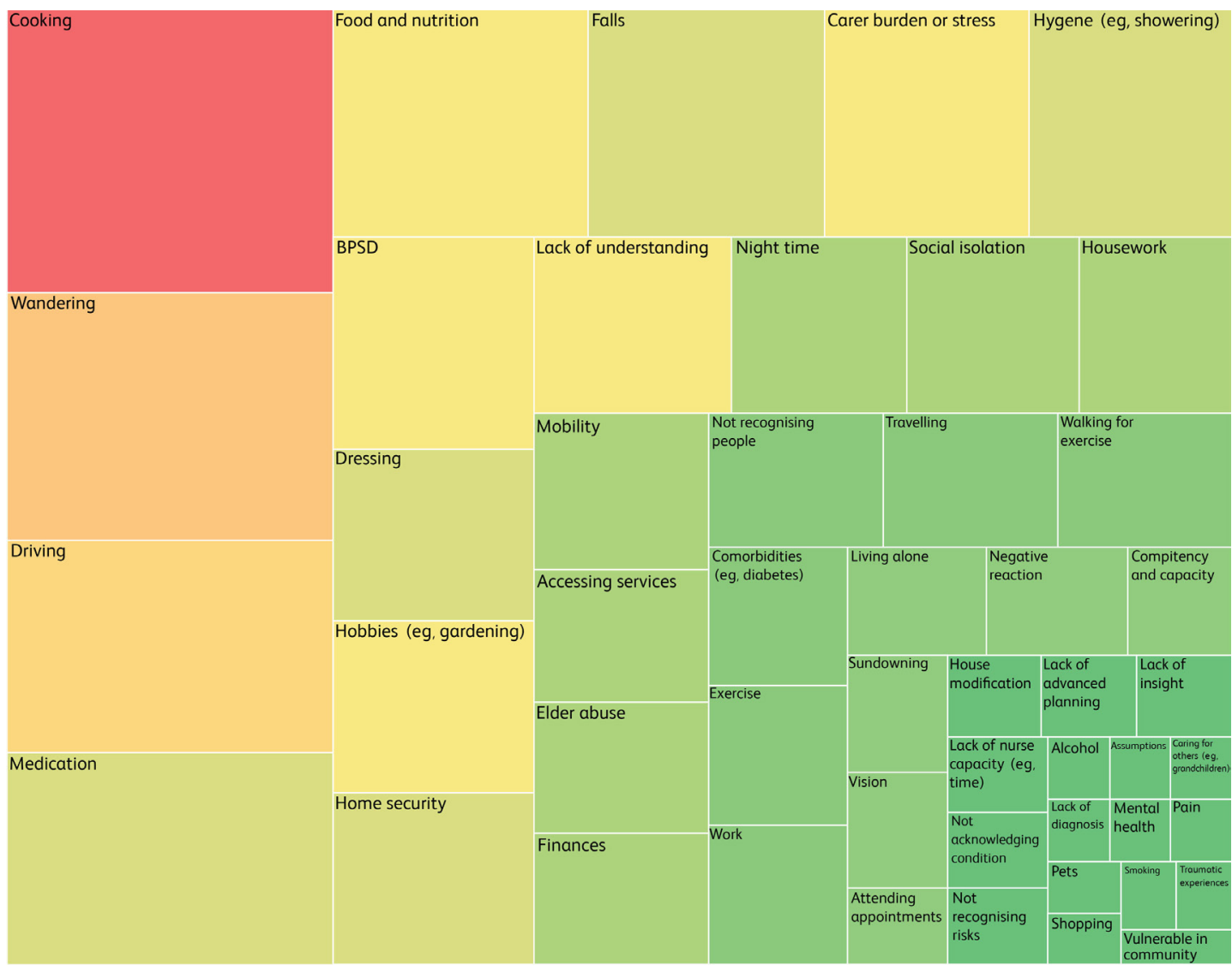

Figure 1 Heat map of identified risks. The size and depth of colour of the boxes in the heat map reflects the number of individuals within our study who identified the risk during their interview. For example, red, orange and yellow depict risks that were discussed most often and green depicts those risks which were discussed less. 
Participants also indicated that the tool needed to be not too confronting but at the same time needing to be honest and authentic:

‘... the tool would be an opportunity to have a discussion with a family member as well. So I wouldn't like to see it too confronting, but at the same time it needs to honest and authentic.' (Staff 004)

'... [when] you administer an MMSE or a RUDAS, people feel like they're being questioned. People feel threatened. So if you're going to do a tool, you've got to really make it a nonthreatening tool. It's almost got to be conversational'(Staff 001)

Because of the constraints on healthcare workers, staff indicated that the tool needed to be user friendly:

‘...you have to make it, obviously, user friendly because nurses are so busy and they have got so much information.

... But it can't be too simple, because then people will miss the point.' (Staff 005)

In addition, the tool needed to be part of the overall assessment process but tailored to the individual:

'As long as it can be tailored to the individual... They should have their own wishes that should be stated in there somewhere. ...It would be good for people ... to be able to say, at what point do you think you might? When you can't do certain things?' (Staff 004)

There was also concern by staff about how the tool would be accessed and whether it would be paper based or electronic:

'...electronic is the way to go because if we stay paper based then we're not keeping up with what the rest of the organisation is doing. ... - I love the face to face aspect' (Staff 007)

It was also queried whether it would provide a clear care plan and links to resources:

'...it's great to have Alzheimer's Australia but to be able to have local resources would be great.' (Staff 012)

Carers identified that it was necessary for a tool to focus on the 'big picture' and people with dementia felt that the tool should focus on abilities and not just deficits and also recognise people with dementia still want independence and autonomy:

'Don't just look at a particular scenario like she can't make a cup of tea, therefore she can't live alone.' (Carer 3)

'I still want independence and autonomy. My husband did used to struggle with that a lot...occasionally I probably selectively choose to forget that I shouldn't cook on my own, because it's frustrating. I've got signs all over the house to say do this, or don't do that, or here's how to do it. I've got lots of lists that just give me the instructions in order for things that used to be automated, but are no longer. I've learned to live with them as humiliating as I used to think and feel they were. I've had to put them in the life enhancement aids category like just the pair of reading glasses...perhaps you have something that helps people see their way to seeing the symptoms of dementia as disabilities and therefore finding strategies to support them.' (Person with Dementia 002)

As it was identified by participants that what one person considered to be a risk may not be considered a risk by someone else, suggestions on how to identify what constitutes a risk to an individual were provided:

'Well I would have a whole lot of cards out with all the pictures on them and say what do you think is a risk?' (HOP (Focus Group 2))

\section{Tool development}

A prototype electronic discussion tool was developed based on the analysis of the interview data and feedback provided by the stakeholder advisory group. The electronic format was chosen as there is a significant shift to electronic records within the healthcare setting. This discussion aid can be completed on a computer or tablet in a home or healthcare setting and is primarily for use by health professionals when discussing the issue of risk with people with dementia and their carers. Acknowledging that not all settings have moved to electronic records a paper-based version of the tool was also created; however, due to its inability to direct the user automatically to only the relevant options, it is less optimal than its electronic counterpart. The discussion tool aims to move from a purely deficit model to one which acknowledges what the person with dementia can do and their preferences moving forward.

Twelve flash cards have been developed for use with the electronic or paper-based version of the discussion tool to assist individuals by prompting issues they may want to discuss with the health professional (see supplementary file 1). The cards feature pictorial images and easy-to-understand English and encompass the major issues facing those living with dementia, including prompts on health, food, grooming and hygiene, finances, socialising and work, leisure activities, chores, mobility, transport, changing behaviours and planning for the future. In addition, there is a final card that invites the individuals taking part to mention any issues that may not fall into any of these categories.

The use of flash cards has number of purposes; it provides a prompt for all parties involved to consider issues that they may not have otherwise, while allowing for those with visual or tactile ways of thinking to engage effectively. All of the cards can be used at once, or placed out one at a time, and the tool can be fully completed over multiple sessions if needed.

The structure of the tool includes:

1. Gathering information about the person with dementia that may inform care (eg, diagnosis, availability of support, pertinent life events, cultural, spiritual or religious group affiliation). This may already be done in other assessments during admission to the service. 
2. Establishing what activities and issues are important to the person with dementia.

3. Establishing issues which the person with dementia, carer or health professional are worried about or that are perceived as becoming more risky.

4. Discussion of who the risk is to, strategies to assist the person with dementia and/or carer and whether or not a referral to a senior nursing specialist or social worker is required.

Each of these four stages is important in order to provide a holistic view of the individual and those around them and come to an appropriate and tailored decision relating to the issue(s) at hand. These four parts are then documented and form part of the care plan for the person with dementia and inform current and future care. The adaptive nature of the online aid means that only those activities or issues which are identified as being worrisome or risky by the person with dementia, carer and/ or health professional are discussed in detail, minimising burden on all involved.

\section{Phase II: Postdevelopment feedback on the tool}

Participants were again asked to provide their feedback on the 'risk negotiation' discussion tool: the use of flash cards to prompt issues that they may not have thought of, the bespoke nature of the electronic tool and how the tool could be amended to make it more user-friendly were all seen as very positive. Every participant who evaluated and provided feedback on the tool indicated that they found it an acceptable tool and that it would be useful in the context of community dementia care. While some suggested improvements to the tool, these were generally of a small nature (eg, rewording, changing colours of cards, adding an additional category). The overall structure and content of the tool was generally responded to positively and the ability to ensure that topics often overlooked were discussed was a particular strength of the tool:

I think it is a very good tool. ... because so many of our clients are being seen because they've got dementia, all these little things will help.... Sometimes there's all these other little things happening around, and you just need sometimes a reminder to just go oh yeah, I haven't even looked in their shower to see if it's actually been used in the past 6 months. But that would probably get prompted by a question.' (RN 007)

The complexity of care for people living with dementia was a further reason that the risk negotiation tool was useful in this context:

'I think it's a great idea. ... when you're in the middle of a complex situation, sometimes you can't see the forest for the trees. So it's really good to be able to have some kind of tool... that just lets you get your head around it.' (Staff 005)

It was also seen as a way to facilitate a holistic discussion about what the situation is and what may be needed:
'I think [it will be useful] as a group discussion with nurses and family so that everybody knows where everybody else is' (Carer 016)

The ability for the tool to tailor support or care goals to the person with dementia, their carers and/or family was also seen as a strength:

It actually gives a good focus in terms of goals and then review of care goals or support goals to actually come back to the life story and more towards what I call a social model rather than a medical model.' (Staff 019)

In particular, participants liked the use of the flash cards as a way to gather more information about the person with dementia and their carer and/or family:

'Yeah, I think with the cards and getting them to pick what's important is probably really good. Because if you just sit there and ask questions it goes in one ear and out the other. Whereas if there's some sort of visual or something, I think it would work a bit better.' (RN 003)

'Yeah. I think it-yeah, because I think prompt cards are great too, because they make you think of-sometimes when you are in there struggling with their behaviours too, it's good to have those little prompts.' (RN 004)

'It's a good prompt... so we remember everything you need to cover. That's what would be typical of most people.' (Person with dementia 003)

In addition, the ability to comprehensively document the conversation was seen as a way to facilitate autonomy for people with dementia by promoting an enablement approach in nurses, who can have a tendency to be risk averse:

'Yes, I can see how that would work. I can see [documenting the discussion is] a really good idea, yes... because a lot of the case-like you say, nurses are risk averse or seem to be risk averse-and a lot of the thing is, if it goes wrong it's my fault. If I wrote it down, not my fault.' (Carer 003)

\section{DISCUSSION}

Our exploration of the issue of risk in people with dementia, carers, HOP, health professionals and health organisations identified a significant number of perceived risks to people with dementia. In contrast to the literature, health professionals and carers in this study had a fairly open view to people with dementia taking acceptable risks. Participants also spoke of risk as a complex issue particularly in relationship to dementia care and that those risks that are experienced need to be addressed in an individualised manner.

The premise of a support aid to facilitate a discussion about risk between people with dementia, carers and health professionals was seen as an acceptable means in which to identify and address individual concerns and a means to develop a care plan that facilitated autonomy and choice for a person with dementia and their carer. 
Information gleaned from the Phase I interviews in regard to risk and about the format and content of a facilitated negotiation tool was used to inform its initial development. Phase II feedback from interviews with consumers and health professionals was then used to inform a refined final 'user-friendly' version of a tool which facilitates a discussion about identifying areas of concern and strategies to address concerns on an individual level in line with participants' aspirations (see supplementary file 2).

The creation of a tool to assist in the determination of risk in regard to persons with dementia is not entirely novel. Manthorpe and Moriaty ${ }^{21}$ received funding from the UK Department of Health in 2009 to develop a framework to encourage everyone involved in supporting persons with dementia to take an enabling and measured approach to risk. Their framework outlines the key issues and evidence in considering risk with people with dementia and provides a structure which practitioners can use to assess, enable and manage risk with persons with dementia and their carers using a scoring system to identify key risks for the person with dementia and others and the likelihood of something bad happening or the severity of the danger. There are also details on how to develop a personal portfolio or 'heat map' for a person with dementia that provides a framework in which to consider each risk and the balance between quality of life. ${ }^{21}$

A further tool recently developed by Pond $e t a t^{22}$ was adapted from Manthorpe and Moriaty's risk assessment framework ${ }^{21}$ for use in the Australian setting. This multifaceted risk assessment tool 'Planning for my future' was designed for use by individuals themselves, to assess and manage current and future physical and psychosocial risks to independence and quality of life while living in the community with the purpose of facilitating, safety and quality of life and not for use by health professionals. ${ }^{22}$

Our tool differs to both that of Manthorpe and Moriarty' $^{{ }^{21}}$ in that it is not designed exclusively for use by health professionals to determine the extent of a risk using a heat map approach and to Pond et als 'Guidance' framework and assessment tool ${ }^{22}$ that enables health professionals and carers and persons with dementia living in the community to assess or consider the issues of risk independent of each other. Our tool is specifically developed, as a discussion aid, to facilitate communication between people with dementia, carers, family members and health professionals together to identify concerns and from whose perspective as well as to jointly develop strategies to address any concerns. The overall purpose of the discussion aid is to enable the wellbeing of people with dementia by providing them with 'choices' while alleviating the concerns of carers/familyand health professionals.

Additionally, although there is evidence that decision support aids can help to involve people in decision-making by providing a way to clarify personal values, risk perception, improve knowledge and provide options, there is little evidence about how to implement them successfully across healthcare settings. ${ }^{23-26} \mathrm{We}$ have overcome this barrier through the development of an electronic discussion tool that can be incorporated into a health service organisations client record system for use as standard care. The logical progression of this developmental study is to test its feasibility and effectiveness in a real-word setting.

There are a number of limitations that must be considered in relation to this research. Despite significant effort, the number of people with dementia recruited to this study was limited; however, appropriate numbers of carers were included which alleviates this concern to a small extent. In addition, the people with dementia who were included in this study tended to be younger and early in the trajectory of their disease. Participants tended to reside in areas that are deemed as being higher socioeconomic status.

There are, however, a number of strengths of this research. Inclusion of codesign and PAR principles ensured that the issue of risk was gained from the perspectives of people with dementia, their carers, HOP, health professionals and healthcare organisations. In addition, feedback on our 'Risk Negotiation' discussion tool deemed that it was acceptable to people with dementia, carers and health professionals.

To our knowledge, this is the first electronic resource that has been codesigned by consumers that enhances health professionals' ability to provide care according to the recently released Clinical Practice Guidelines and Principles of Care for People with Dementia ${ }^{27}$ by facilitating the delivery of 'Person-centred care', identifying and responding to the individual needs and preferences of the person with dementia, their carer and family.

\section{CONCLUSION}

Our codesigned discussion tool enables choices for people with dementia by focusing on abilities rather than deficits and assists health professionals to deliver person-centred care. Flash cards prompt concerns and the electronic tool, provides a range of strategies to address these issues. We recommend that the tool now be trialled in a real life setting.

\section{Implications for practice}

Our 'Risk Negotiation' tool provides health professionals with a format to gain a holistic view of an individual and those around them, establish what their needs and concerns are and provide strategies and a documented care plan that enhances the choices of people living with dementia. Facilitation of a discussion about concerns and strategies to address these and the documentation of both may alleviate health professionals' concerns about breaching perceived duty of care.

\section{Author affiliations}

${ }^{1}$ RDNS Research Institute, Royal District Nursing Service, St Kilda, Victoria, Australia ${ }^{2}$ Faculty of Medicine and Public Health, The University of Newcastle, Callaghan, New South Wales, Australia

${ }^{3}$ NHMRC Cognitive Decline Partnership Centre, Sydney Medical School, Northern, The University of Sydney, St Leonards, New South Wales, Australia

${ }^{4}$ Central Clinical School, Monash University, Clayton, Victoria, Australia

${ }^{5}$ Primary Care for Older People, University College London, London, UK 
${ }^{6}$ School of Medicine and Public Health (General Practice), The University of Newcastle, Callaghan, New South Wales, Australia

Acknowledgements The authors acknowledge the contribution of consumers, staff and all members of the project Advisory Group and that Home and Community Care (HACC). Services provided by RDNS are jointly funded by both the Victorian and Australian Governments. They also acknowledge the Victoria Bowls Association members who participated in the focus groups, Ms Emma Renehan for her assistance at one of these focus groups and the contribution of Ms Louise Bellizzi, Senior Clinical Nurse Advisor, for sharing her expert knowledge.

Contributors DPG conceived and initiated the study. MD, DPG, FOK, SI and DP developed the 'Risk Negotiation' decision aid with feedback and suggestions from the project Advisory Group members. MD, DPG and F0 undertook the data collection. MD and DPG undertook the data analysis and final drafting of the article and revised it for critical content. All authors contributed to and approved the final version of the paper and accept accountability for all aspects of the work.

Funding Funding for this study was provided by State Trustees Australia Foundation.

Disclaimer We confirm that all personal identifiers have been removed or disguised so that participants described are not identifiable.

Competing interests None declared.

Ethics approval RDNS Human Research Ethics Committee (Approval number 154).

Provenance and peer review Not commissioned; externally peer reviewed.

Data sharing statement There are no additional published data from the study.

Open Access This is an Open Access article distributed in accordance with the Creative Commons Attribution Non Commercial (CC BY-NC 4.0) license, which permits others to distribute, remix, adapt, build upon this work non-commercially, and license their derivative works on different terms, provided the original work is properly cited and the use is non-commercial. See: http://creativecommons.org/ licenses/by-nc/4.0/

(c) Article author(s) (or their employer(s) unless otherwise stated in the text of the article) 2017. All rights reserved. No commercial use is permitted unless otherwise expressly granted.

\section{REFERENCES}

1. World Health Organisaton and alzheimer's disease international. Dementia: a public health priority. Geneva, Switzterland: World Health Organisation, 2012.

2. Australian institute of health and welfare. Dementia in Australia. Cat. no. AGE 70. Canberra: AlHW, 2012.

3. Hussein S, Manthorpe J. The dementia social care workforce in England: secondray analysis of a national workforce dataset. Aging Ment Health 2012;16:110-8.

4. I SK, Kirkevold M, Engedal K. Ethical dilemmas concerning autonomy when persons with dementia wish to live at home: a qualitative, hermeneutic study. BMC Health Service Research 2016:16:1-12.

5. Bailey C, Clarke CL, Gibb C, et al. Risky and resilient life with dementia: review of and reflections on the literature. Health Risk Soc 2013;15:390-401.

6. Universal Declaration of Human Rights, United Nations General Assembly, Paris, 1948. http://www.un.org/en/universal-declarationhuman-rights/index.html (accessed 8 Mar 2017)
7. Australian Aged Care Act 1997. Australian Residential Aged Care Charter of Residents' Rights and Responsiblities. http://www.sa. agedrights.asn.au/residential_care/the_charter_of_residents_rights_ and_responsibilities (accessed 8 Mar 2017).

8. Powers of Attorney Act 2014. Office of the public advocate. http:// www.publicadvocate.vic.gov.au/assessing-whether-a-person-hasdecision-making-capacity (accessed 13 Feb 2017).

9. Mitchell G, Agnelli J. Person-centred care for people with dementia: Kitwood reconsidered. Nurs Stand 2015;30:46-50.

10. Ibrahim JE, Davis MC. Impediments to applying the "dignity of risk" principle in residential aged care services. Australian J Ageing 2013;32:188-93.

11. Manthorpe J, Samsi K. Person-centred dementia care: current perspectives. Clinical Interventions in Ageing 2016;11:1733-40.

12. Department of Health. Living well with dementia: a National dementia strategy. London: Department of Health; 2009.

13. Banerjee S. Living well with dementia-development of the national dementia strategy for England. Int J Geriatr Psychiatry 2010;25:917-22.

14. Bowling A. Aspirations for older age in the 21st century: what is successful aging? Int J Aging Hum Dev 2007;64:263-97.

15. St John P, Montgomery P. Cognitive impairment and life satisfaction for older adults with dementia in the US health care system. J Geriatr Psychiatry 2010;25:814-21

16. Yost J, Dobbins M, Traynor R, et al. Tools to support evidenceinformed public health decision making. BMC Public Health 2014;14:728. http://www.biomedcentral.com/1471-2458/14/728

17. The Kings Fund. http://www.kingsfund.org.uk/projects/ebcd/ experience-based-co-design-description (accessed 11 Apr 2017).

18. Friere K, Sangiorgi D. Service design and healthcare innovation: from consumption to co-production and co-creation. Proceedings of the 2nd Nordic Conference on Service Design and Service Innovation 2010. Linkoping, Sweden. http://www.servdes.org/pdf/2010/freiresangiorgi.pdf (accessed July 2017).

19. Braun V, Clarke V. Using thematic analysis in psychology. Qual Res Psychol 2006;3:77-101.

20. NVIVO11 Qualitative solutions and research. Doncaster, Victoria, Australia, 2016

21. Manthorpe J, Moriaty J. "Nothing venture, nothing gained": risk guidance for people with Dementia. Department of Health. www.dh. gov.uk/publications

22. Pond D, Scott R, Day J, et al. Living with Dementia in the community: planning for my future: final report,. Cognitive Decline Partnership Centre 2016.

23. Stacey D, Légaré F, Col NF, et al. Decision aids for people facing health treatment or screening decisions. Cochrane Database Syst Rev 2014:CD001431. http://onlinelibrary.wiley.com/doi/

24. Da Silva D. Helping people share decision making a review of the evidence considering whether shared decision making is worthwhile. The Health Foundation, 2012

25. Elwyn G, Scholl I, Tietbohl C, et al. "Many miles to go ...": a systematic review of the implementation of patient decision support interventions into routine clinical practice. BMC Med Inform Decis Mak 2013;13:S14.

26. Bunn F, Goodman C, Manthorpe J, et al. Supporting shared decisionmaking for older people with multiple health and social care needs: a protocol for a realist synthesis to inform integrated care models. BMJ Open 2017; 7:e014026.

27. Guideline Adaption Committee. Clinical practice guidelines and principles of care for people with Dementia. Sydney: NHMRC Partnership Centre for Dealing with Cognitive and RelatedFunctional Decline in Older People (CDPC), 2016. 